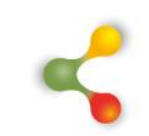

REVUE HYBRIDE DE L'ÉDUCATION

\title{
Éditorial
}

\section{Faire sa place}

Marie-Pierre Baron Directrice de la RHÉ, professeure à l'Université du Québec à Chicoutimi

rhe@uqac.ca 


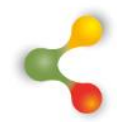

\section{REVUE HYBRIDE DE L'ÉDUCATION}

\section{Avant propos}

Faire sa place... ou « Comment être singulier dans une offre plurielle? » si on reprend le titre de l'article d'opinion que je cosigne avec mes collègues Ugo Collard-Fortin et Marie-Hélène Bruyère. Tel est le défi de la publication scientifique. L'offre est grande dans le monde de la publication francophone en sciences de l'éducation et l'accès en ligne au contenu des revues scientifiques rend certainement la compétition plus grande, mais surtout, permet le partage du contenu au-delà des frontières réelles et créées. Certes, l'accès instantané aux écrits et le nombre grandissant de publications marquent un tournant dans le monde scientifique, mais ils poussent également les revues à aller toujours plus loin pour se démarquer. On en vient donc à se demander comment faire sa place dans tant d'abondance ?

La RHÉ existe depuis 2016 et nous avons fait le pari que nous pourrions tirer notre épingle du jeu en offrant une plateforme et un contenu exclusif et inclusif pour que chercheur-es, chercheur-es en formation, praticien-nes et praticien-nes en formation puissent partager et avoir accès non seulement aux connaissances issues de la recherche, mais aussi à celles issues de la pratique. II a fallu, pour rendre viable ce projet, mettre en place différents dispositifs de promotion et solliciter l'aide d'un réseau de contacts qui s'agrandit constamment. Aujourd'hui, à force de travail, il est possible de penser que nous avons su nous tailler une place, mais tout est encore à faire pour la conserver. II importe cependant de jeter un coup d'œil vers l'arrière pour mieux nous projeter vers l'avant.

La RHÉ est jeune, certes, mais la publication d'un numéro régulier à la fin de décembre 2018 est l'occasion de faire le bilan de ces deux dernières années ou de ses deux premières années! En 2017, un numéro thématique de lancement sur La transformation des pratiques en collaboration avec les milieux scolaires du Saguenay-Lac-Saint-Jean depuis la fin des années 1990 a été mis en ligne. Ce numéro a permis de marquer l'orientation de la RHÉ et de mettre en lumière la recherche participative et collaborative, un créneau fort développé à l'Université du Québec à Chicoutimi.

L'année 2018, pour sa part, aura permis la publication de deux numéros réguliers. Au total dans ces numéros, 6 articles ont été écrits par des chercheurs universitaires (éditoriaux, article d'opinion et article de la rubrique Milieu de recherche), 2 articles ont été écrits par des chercheurs en formation (rubrique Place à la relève scientifique) et 5 articles sont issus de la pratique enseignante (rubrique Milieu de pratique). Ce portrait des numéros réguliers permet de rendre compte que non seulement la RHÉ s'autoproclame inclusive, mais elle l'est réellement. Elle a permis pratiquement à parts égales de diffuser des contenus issus de la recherche et de la pratique. 


\section{6}

\section{REVUE HYBRIDE DE L'ÉDUCATION}

Il importe également de s'attarder aux chiffres, soient aux statistiques de consultation de la RHÉ depuis son hébergement sur la plateforme de publication OJS-3. En somme, pour le premier numéro thématique et le premier numéro régulier, on dénombre 6972 consultations des résumés des articles et 4167 téléchargements des articles publiés. Les statistiques sont impressionnantes pour une jeune revue créée et hébergée en région et l'équipe scientifique ne peut que s'en enorgueillir. II ne faut toutefois pas oublier que ces chiffres en cachent beaucoup d'autres: un nombre incalculable d'heures pour la création, l'édition et le financement de la RHÉ; un nombre tout aussi important d'heures de la part des auteurs non seulement pour écrire les articles, mais aussi pour réaliser les projets pédagogiques et de recherche desquels sont issus ces articles; un nombre de collaborateurs sans cesse grandissant qui assurent l'écriture, mais aussi la révision du contenu, etc.

Le bilan de nos activités est positif et l'année 2019 s'avère tout aussi prometteuse. La RHÉ accueillera des rédacteurs invités pour la publication de numéros thématiques variés qui permettront de mettre en lumière le travail de tous les acteurs de l'éducation. De plus, le changement de gouvernement au Québec amène aussi à croire que l'importance de la diffusion des connaissances issues de la recherche et de la pratique pourra être mise en lumière sur la place publique. La Coalition Avenir Québec présente l'éducation comme étant au sommet des priorités avec des promesses telles que la création des prématernelles pour les enfants de 4 ans, qui nécessiteront de soutenir la formation continue des enseignant-es en exercice, ainsi que celle des futur-es enseignant-e-s. Ainsi, espérons que les différentes plateformes de diffusion seront aussi soutenues dans le but de leur permettre de remplir leur rôle de "passeurs de connaissances", ce rôle joué dans l'espoir de soutenir la réussite éducative de tous les élèves, ainsi que celle des enseignant-es, et ce, pour que chacun puisse à son tour faire sa place...

\section{Aperçu des contributions}

Le deuxième numéro régulier de 2018 est à l'image de la RHÉ. II débute avec trois articles de la rubrique Milieu de recherche. Dans le premier, les professeures-chercheures Élisabeth Jacob et Annie Charron, spécialisées en éducation préscolaire, présentent un article où elles se penchent sur les enjeux d'une recherche collaborative menée avec trois enseignantes atikamekws. Pour sa part, le professeur-chercheur et politicologue Jean Bernatchez discute, dans son article, des instances régionales de concertation sur la persévérance scolaire et la réussite éducative. Finalement, les professeures-chercheures Nicole Monney, Monique l'Hostie et Sylvie Fontaine proposent un article s'intéressant à la formation initiale à l'enseignement. Elles y réfléchissent sur le poids des représentations sociales dans le développement professionnel des futurs enseignants. 


\section{$\&$}

\section{REVUE HYBRIDE DE L'ÉDUCATION}

Ce deuxième numéro régulier propose également deux articles de la rubrique Milieu de pratique. Ces articles ont été écrits à la suite d'entrevues réalisées par des étudiants en formation à l'enseignement préscolaire et primaire auprès de praticiens. Dans un premier article, Patrick Giroux, Jean-Frédéric Girard et Maryka Gagnon présentent une initiative réalisée dans une classe de la commission scolaire des Rives-du-Saguenay où l'enseignant de $6^{\mathrm{e}}$ année utilise la programmation et la robotique. Le second article de Marie-Élaine Gignac, Joanie Desgagné et Nicole Monney porte, pour sa part, sur l'apprentissage, par le développement d'habiletés en lecture et en écriture, de deux langues simultanément.

Le numéro se poursuit avec un article de la rubrique Place à la relève écrit par Louise Savard, étudiante à la maitrise en éducation, et Manon Doucet professeure-chercheure. Dans cet article théorique exposant une problématique de recherche, les auteures présentent et contextualisent une approche éducative novatrice pour outiller les enfants vivant avec de l'embonpoint ou l'obésité.

Finalement, cette deuxième édition de 2018 se termine avec un article " hors série ». II s'agit d'un article d'opinion intitulé Les revues scientifiques en science de l'éducation : comment être singulier dans une offre plurielle? cosigné par Ugo Collard-Fortin, Marie-Pierre Baron et Marie-Hélène Bruyère. II s'agit d'une réflexion au sujet de la publication et des défis contemporains de l'édition scientifique.

\section{Remerciements}

La publication du deuxième numéro régulier de la RHÉ a été possible grâce à la contribution des différents auteurs, des invités assurant l'évaluation du contenu, ainsi que des membres du comité scientifique. Encore une fois, merci à monsieur Ugo Collard-Fortin qui, à titre de coordonnateur, a su rendre la mise en ligne de ces publications possible. Merci également à mesdames Marie-Élaine Gignac et Catherine Vachon qui ont assuré le soutien technique et la révision linguistique des textes. 